\title{
Diagnostic Role of Adenosine Deaminase (ADA) among Tuberculous Meningitis Patients
}

\author{
ABUL KALAM MOHAMMED SHOAB ${ }^{1}$, MOHAMMAD SHAH JAHIRUL HOQUE CHOWDHURY², \\ DEVENDRA NATH SARKAR ${ }^{3}$, MD ISMAIL HOSSAIN ${ }^{4}$, MUHAMMAD AKHTARUZZAMAN ${ }^{5}$, TAKIB \\ UDDIN AHMED ${ }^{6}$, MD TAUHIDUL ISLAM CHOWDHURY ${ }^{2}$, UTTAM KUMAR SHAHA ${ }^{7}$, NARAYAN \\ CHANDRA KUNDU $^{8}$, AMINUR RAHMAN $^{9}$, MASUD RANA $^{10}$, FIROZ AHMED QUARISHI $^{11}$
}

\begin{abstract}
:
Background: In the developing countries where TB is endemic; an ideal test for tuberculous meningitis should be economic, minimally invasive, of high accuracy and quick to perform. In many countries, also in India, several studies were conducted to establish the ADA activity as a sensitive and specific test of tuberculous meningitis. So it is very much needed to evaluate the diagnostic role of CSF ADA in tuberculous meningitis in Bangladesh. Aim: This study aimed to find out CSF ADA as a sensitive and specific test for early diagnosis of tuberculous meningitis. Methods: This case control study was carried out in the Department of Neurology, SSMC and Mitford Hospital, Dhaka from June 2011 to July 2012, to evaluate ADA activity in CSF for diagnosis of tuberculous meninigitis. Results: In the present study, sixty meningitis patients were enrolled. Of which, 30(50\%) were tuberculous meningitis (TBM) taken as cases and rest 30(50\%) were non-tuberculous meningitis (NTBM) taken as control. The CSF ADA activity from TBM patients was compared with CSF ADA from non-TBM infectious meningitis patients. The mean CSF ADA activity was found to be significantly higher in CSF of TBM patients, $14.01 \pm 12.4$ (1.0-65.2), mean $\pm S D$ with range, than in the CSF from non-TBM infectious meningitis, $7.2 \pm 8.2$ (1.8-49.1) $P=0.01$.A cut-off value of $>7.6 \mathrm{U} / \mathrm{L}$ for the TBM patients was calculated from the mean $\pm S D$ of the non-TBM patients. The ADA sensitivity is $81.82 \%$, specificity $65.31 \%$, accuracy $68.33 \%$, PPV $34.62 \%, N P V \quad 94.12 \%$, positive likelyhood ratio 2.3 and lastly negative likelyhood ratio 0.2 for infectious TBM when this cut-off value was used.ROC curve shows area under curve of .736 suggests a moderate accuracy of the test in detection of tuberculous meningitis. Conclusion: This study demonstrated that ADA activity in the CSF of TBM patients, using a cut off value $7.6 \mathrm{U} / \mathrm{L}$, can be useful for the early differential diagnosis of TBM. This test can be performed in any pathology laboratory where more sophisticated methods are not available.
\end{abstract}

\section{Introduction:}

Tuberculosis is one of the leading causes of mortality and morbidity in developing countries. The $\mathrm{WHO}$ reports puts to the record that globally, approximately 16 million people are suffering from active TB with an estimated 8.5 million developing active TB each year, resulting in approximately 2 million deaths ${ }^{1}$. Tuberculous meningitis (TBM) is a

1. Consultant (Medicine), Upazilla Health Complex, Rajnagar, Moulvibazar, Bangladesh.

2. Assistant Professor, Dept. of Neurology, National Institute of Neurosciences \& Hospital, Sher-e- Bangla Nagar, Dhaka, Bangladesh.

3. Associate Professor, Department of Medicine, Rangpur Medical College \& Hospital, Rangpur.

4. Registrar, Department of Medicine, Rangpur Medical College \& Hospital, Rangpur.

5. Registrar, Department of Cardiology, Bangladesh Medical College \& Hospital, Dhaka.

6. Associate Prof. of Neurology, SSMCH\&MH, Mitford, Dhaka.

7. Associate Professor of Neurology, National Institute of Neurosciences \& Hospital, Sher-E-Bangla Nagar, Dhaka, Bangladesh.

8. Associate Prof. of Neurology, ShSMCH, Sher-e-Bangla Nagar, Dhaka.

9. Registrar, Dept. of Neurology, SSMCH\&MH, Mitford, Dhaka.

10. Medical Officer, Dept. of Neurology, BSMMU, Dhaka.

11. Professor of Neurology, National Institute of Neurosciences \& Hospital, Sher-E- Bangla Nagar, Dhaka, Bangladesh. 
common infectious disease of the central nervous system in developing countries. Early diagnosis and treatment with chemotherapy and active management of the complications are of great importance to prevent the irreversible neurologic sequele and death. Delay in diagnosis and so in the start of effective treatment results in poor prognosis and sequalae in up to $25 \%$ of cases ${ }^{2}$. A definitive diagnosis of tuberculous meningitis depends on identifying Mycobacterium tuberculosis in the cerebrospinal fluid (CSF) by direct staining or culture. However, the diagnostic yield of CSF smears and cultures has been very low ${ }^{3}$, and mycobacterial culture may take up to 6 weeks to yield results. Therefore, the diagnosis of tuberculous meningitis depends on the clinical manifestations of subacute to chronic meningitis with lymphocytic CSF and low CSF glucose levels. However, other forms of meningitis may mimic tuberculous meningitis. Certain patients with tuberculous meningitis may have CSF findings resembling aseptic meningitis. Several tests for the rapid diagnosis of tuberculous meningitis have been developed; all are based on examination of the CSF. These tests are considered indirect tests (usually measuring a product of the host response to his infection, such as adenosine deaminase(ADA), the radioactive bromide partition test and antibodies to the mycobacterial antigen) and direct tests usually measuring a product of the infecting organism, such as 3-(2'-ketohexyl) indoline, detecting of tuberculostearic acid (a component of the cell wall of M. tuberculosis), mycobacterial antigens or fragments of mycobacterial DNA by polymerase chain reaction ${ }^{4}$. These methods except adenosine deaminase are too complicated or expensive for many laboratories. Adenosine deaminase (ADA) is an enzyme involved in purine catabolism. It is considered as an indicator of cell-mediated immunity and is found mainly in $T$ lymphocytes ${ }^{5}$. TBM is the severe form of extrapulmonary tuberculosis occurring in 7.0-12.0\% of TB patients in developing countries with high rate of mortality due to delay in diagnosis and proper treatment ${ }^{6}$. In the absence of an early diagnosis and treatment, tuberculous meningitis is characterized by high mortality (20-50\%) and morbidity $(20-30 \%)^{7}$.

The cytological and biochemical analysis of cerebrospinal fluid is the cornerstone for diagnosis but there are diagnostic difficulties many a times in differentiating tuberculous meningitis from nontubeculous meningitis. A gold standard for diagnosis of TBM is an identification of Mycobacterium tuberculosis in cerebrospinal fluid (CSF) by direct smear and culture.

Adenosine deaminase (ADA) is an enzyme with principal biological activity in $\mathrm{T}$ lymphocytes. It is required for lymphocyte proliferation and differentiation. The enzyme activity is known to be elevated in certain infection where immunity is cell mediated like in CSF of TBM patients ${ }^{8-10}$. Various studies have been conducted demonstrating CSF ADA estimation as an enzymatic assay in diagnosis of Tuberculous meningitis and can differentiate TBM from normal subjects or other infectious meningitis.

\section{Materials and Methods:}

It is a case control study. Thirty cases of tuberculous meningitis and thirty non-tuberculous meningitis cases were studied. All eligible subjects as per inclusion and exclusion criteria coming to the Department of Neurology and Medicine, Sir Salimullah Medical College \& Mitford Hospital,Dhaka Medical College Hospital and Bangabandhu Sheikh Mujib Medical University from July 2011 to June 2012 were included till desired sample size was reached. The laboratory works were performed in the department of microbiology \& immunology, BSMMU and Sir Salimullah Medical College, Dhaka.Sampling method was purposive and all meningitis patients seeking treatment were enrolled in the study as per following inclusion and exclusion criteria. They were of either sex, and age range was from fifteen years and above. Diagnosis of tuberculous meningitis were based on presence of clear sign and symptoms of TBM or presence of AFB in CSF. ZN stainining and /or culture positivity for AFB or good response to antituberculous drugs. The meningitis patients who failed to fulfill the above criteria were considered as nontuberculous meningitis and were taken as controls.

CSF ADA was carried out in all the patients of both tuberculous and non-tuberculous meningitis as per exclusion and inclusion criteria. Three CSF sample were collected, first was for cytological test, biochemical and microbiological test and second for CSF ADA and third was for CSF AFB culture. Two milliliters of CSF was sent for ADA assay.

\section{Statical Analysis:}

Among the meningitis patients tuberculous group were considered as cases and non tuberculous 
group were considered as control. CSF ADA levels were compared between cases \& controls. Data were compiled and analyzed by using SPSS version 16.0. Cut off value of ADA level for the diagnosis of tuberculous meningitis was done by using receiver operating characteristic curve (ROC) in the same version of SPSS. Student t- test was used for test of significance, $p<0.05$ was considered as statistically significant.

\section{Results:}

Table-I

Distribution of the respondents by age and sex

\begin{tabular}{lcc}
\hline & Frequency & Percentage \\
\hline Age in years & & \\
$15-20$ & 7 & 11.7 \\
$20-39$ & 32 & 53.3 \\
$40-49$ & 10 & 16.7 \\
$>=50$ & 11 & 18.3 \\
Sex & & \\
Male & 34 & 56.7 \\
Female & 26 & 43.3 \\
\hline
\end{tabular}

Table-I shows the distribution of the study subjects by age and sex. Among the subjects $11.7 \%$ were aged below 20 years, $53.3 \%$ were aged between 20 - 39 years, $16.7 \%$ were aged between 40 - 49 years and $18.3 \%$ were aged above 50 years. Regarding sex $56.7 \%$ were male and $43.3 \%$ were female.

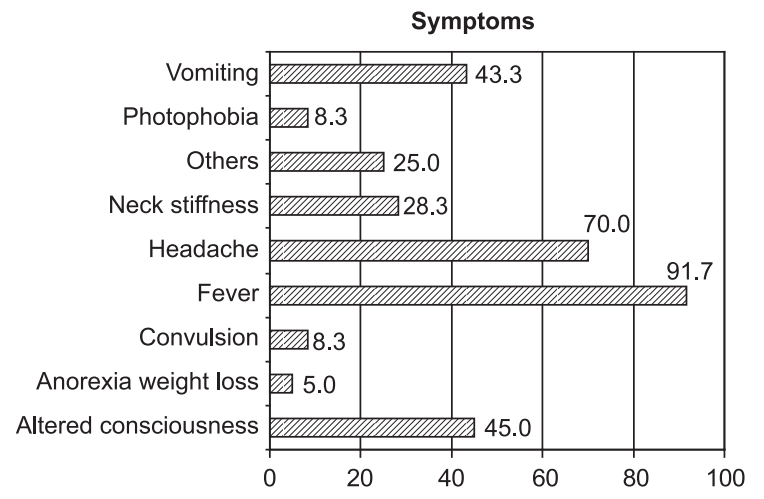

Fig.-1: Distribution of the study subjects by presenting symptoms

Figure-1 depicts the distribution of the study subjects by presenting symptoms. Among them most prevalent symptom was fever (91.7\%). Among others, headache $(70 \%)$, altered consciousness (45\%), vomiting (43.3\%) and neck stiffness $(28.9 \%)$ were notable.
Table-II

Distribution of the study subjects

by physical sign

\begin{tabular}{lcc}
\hline Physical sign & Frequency & Percentage \\
\hline Neck Rigidity & 57 & 95.0 \\
Anemia & 33 & 55.0 \\
Kernig's sign & 28 & 46.7 \\
Long tract sign & 12 & 20.0 \\
Cranial Nerve Palsy & 6 & 10.0 \\
\hline
\end{tabular}

Table II shows the distribution of the study subjects by physical sign. Among the study subjects $55.0 \%$ had anemia, $95.0 \%$ had neck rigidity, $10.0 \%$ had cranial nerve palsy, $46.7 \%$ had kernig's sign and $20.0 \%$ had long tract sign.

Table-III

Distribution of the study subjects by fundoscopic examination findings

\begin{tabular}{lcc}
\hline Findings & Frequency & Percentage \\
\hline Normal & 50 & 83.3 \\
Papilloedema & 8 & 13.3 \\
Optic atrophy & 2 & 3.3 \\
\hline Total & 60 & 100.0 \\
\hline
\end{tabular}

Table III shows the distribution of the study subjects by fundoscopic examination findings, where 83.3 $\%$ had normal fundus, $13.3 \%$ had papilloedema and $3.3 \%$ had optic atrophy.

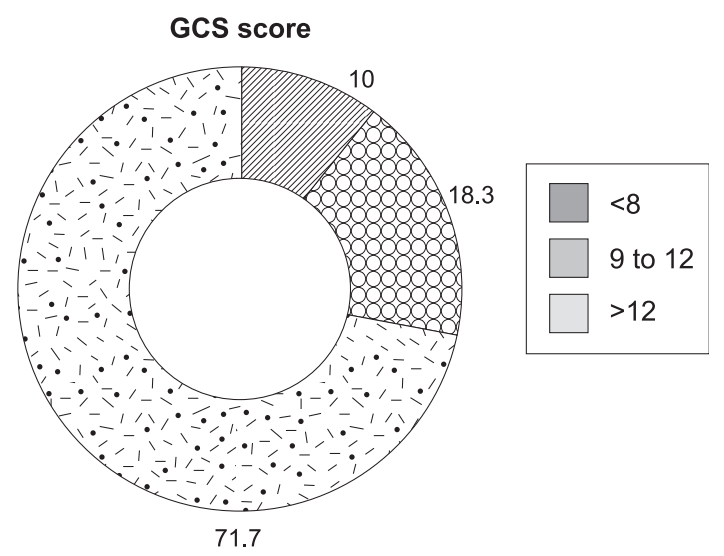

Fig.-2: Distribution of the study subjects by GCS score

Figure 2 shows the distribution of the study subjects by GCS score. Among the study subjects $10.0 \%$ had GCS score less than $8,18.3 \%$ had between 9 -12 and $71.7 \%$ had over $>12$. 
Table -IV

Different parameters of CSF in various Meningitis

\begin{tabular}{|c|c|c|c|c|c|c|}
\hline & \multicolumn{2}{|c|}{ Tuberculous $(n=30)$} & \multicolumn{2}{|c|}{ Bacterial $(n=12)$} & \multicolumn{2}{|c|}{$\operatorname{Viral}(n=18)$} \\
\hline & Mean $\pm S D$ & Range & Mean $\pm S D$ & Range & Mean $\pm S D$ & Range \\
\hline Sugar (mmol/L) & $2.6 \pm 1.2$ & $0.8-6.5$ & $2.606 \pm 1.2$ & $0.4-4.5$ & $4.01 \pm 1.1$ & $2.1-6.6$ \\
\hline Protein (mg/dl) & $212.3 \pm 275.1$ & $37-1440$ & $142.1 \pm 99.7$ & $66-388$ & $73.8 \pm 55.2$ & $21-226$ \\
\hline TC (/cmm) & $484.7 \pm 1317$ & $5-6400$ & $192.4 \pm 199.9$ & $5-840$ & $27.24 \pm 48.3$ & $0-180$ \\
\hline lymphocyte(\%) & $72.08 \pm 31.2$ & $10-100$ & $31.25 \pm 36.8$ & $5-100$ & $88.24 \pm 23.4$ & $10-100$ \\
\hline Polymorph(\%) & $28.12 \pm 31.6$ & $0-95$ & $68.75 \pm 37.5$ & $0-95$ & $12.1 \pm 6.47$ & $0-44$ \\
\hline
\end{tabular}

Table IV shows the different parameters of CSF in various meningitis. In TBM, sugar was low $2.6 \pm 1.2$ (range $0.8-6.5$ ), protein was high $212.3 \pm 275$. (range 137 - 1440), total leucocytes count was high $484.7 \pm$ 1317 (range 5-6400) and lymphocytic pleocytosis $72.08 \pm 31.2$ (range $10-100$ ). In bacterial meningitis, sugar was low $2.606 \pm 1.2$ (range $0.4-4.5$ ), protein was high 142.1 99.7 (range 66 - 388), total cell count was high $192.4 \pm 199.9$ (range $5-840$ ) and polymorph was $68.75 \pm 37.5$ (range $0-95$ ). In viral meningitis, sugar was normal $4.01 \pm 1.1$ (range 2.1 -6.6 ), protein was mildly high $73.8 \pm 55.2$ (range 21 - 226 ), cell count was high $27.24 \pm 48.3$ (range 0 180 ) mostly lymphocytes $88.24 \pm 23.4$ (range 10 100).

Table -V

Distribution of the Laboratory findings

\begin{tabular}{lcc}
\hline & Frequency & Percentage \\
\hline Gram Staining $(\mathrm{n}=60)$ & & \\
Positive cocci & 2 & 3.3 \\
Negative cocci & 58 & 96.7 \\
MT $(\mathrm{n}=30)$ & & \\
$<10 \mathrm{~mm}$ & 17 & 56.3 \\
$>=10 \mathrm{~mm}$ & 13 & 43.7 \\
\hline
\end{tabular}

Table $\mathrm{V}$ shows the distribution of the laboratory finding. Among the study subjects 3.3\% showed gram positive cocci in gram stain. And $43.7 \%$ showed MT over $10 \mathrm{~mm}$. thirty subjects did not undergo MT as they were not the suspected TBM cases.

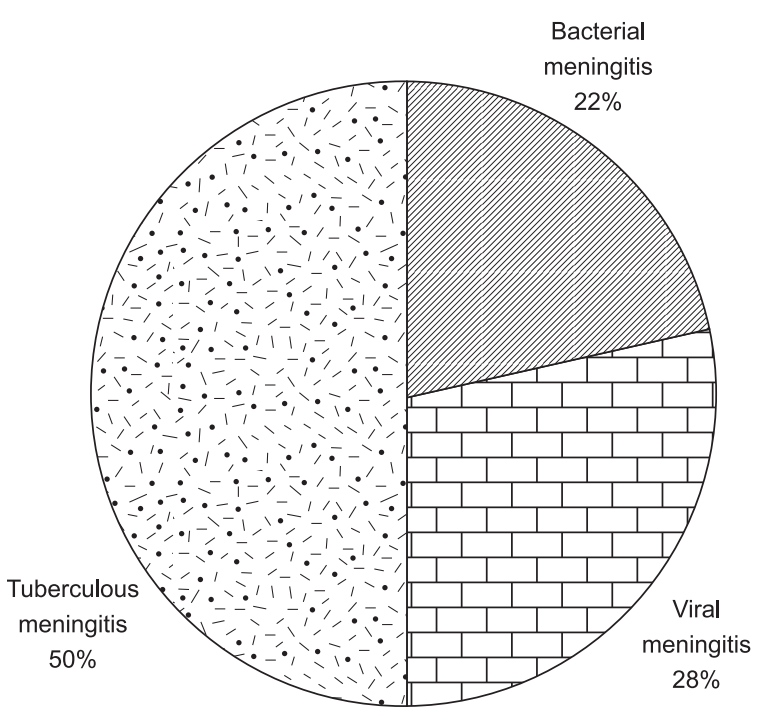

Fig.-3: Distribution of the study subjects by diagnosis

Sixty meningitis patients were enrolled in the study, of which, $50 \%(30)$ were tuberculous meningitis(TBM) and rest $50 \%$ (30) were nontuberculous meningitis(NTBM). Among the NTBM, $28 \%$ cases were viral meningitis and $22 \%$ cases were bacterial meningitis(Fig.3).

Table -VI

CSF ADA level in comparision to CSF AFB culture and sensitivity $(c / s)$ findings

\begin{tabular}{lccc}
\hline CSF AFB C/S & $\begin{array}{c}\text { CSF ADA(U/L) } \\
\text { Mean } \pm S D\end{array}$ & Range(U/L) & P value \\
\hline Positive $(n=11)$ & $13.42 \pm 6.76$ & $4.7-28.9$ & 0.35 \\
Negative $(n=49)$ & $9.97 \pm 11.69$ & $1.0-65.2$ & \\
\hline
\end{tabular}


Table VI shows mean CSF ADA level. In AFB positive cases, it was 13.42 \pm 6.76 (range 4.7-28.9) and in AFB negative cases it was 9.9 11.69 (range 1.065.2). ADA level was higher in AFB positve cases.

Table-VII

CSF ADA result in various group of meningitis

\begin{tabular}{lccc}
\hline Group & $\begin{array}{c}\text { Mean } \\
\text { ADA (U/L) }\end{array}$ & SD & $\begin{array}{c}\text { Range of ADA } \\
(\mathrm{U} / \mathrm{L})\end{array}$ \\
\hline TBM $(\mathrm{n}=30)$ & & & \\
Confirmed cases (11) & 13.4 & 6.76 & $4.7-28.9$ \\
Probable cases (19) & 14.3 & 14.9 & $1.0-65.2$ \\
Total (30) & 14.01 & 12.4 & $1.0-65.2$ \\
Bacterial meningitis (n-12) & & & \\
Confirmed cases (n-2) & 6.2 & 1.1 & $5.4-7$ \\
Probable cases $(\mathrm{n}-10)$ & 11.2 & 19.2 & $2.0-49.1$ \\
Total (n-12) & 10.4 & 12.4 & $2.0-49.1$ \\
Viral meningitis $(\mathrm{n}=18)$ & 5.09 & 1.92 & $1.8-8$ \\
NTBM(n=30) & 7.2 & 8.2 & $1.8-49.1$ \\
\hline
\end{tabular}

In TBM ADA was positive in 20 cases and the mean was 18.3U/L (range 8.7-65.2U/I), ADA was negative in 10 cases and the mean was $5.34 \mathrm{U} / \mathrm{L}$ (range 1.07.0u/l). However, the overall result of ADA in TBM group was 14.01U/L \pm 12.4 , (range 1.0-65.2 U/L). In bacterial meningitis the ADA result was $10.4 \mathrm{U} /$ $L \pm 12.2$, (range 2.0-49.1 U/L) and in viral meningitis 5.09U/L \pm 1.92 , (range 1.8-8 U/L).In NTBM ADA positve was in 6 cases and the mean was 15.9U/L (range 8-49.1), ADA negative was in 24 cases and the mean value was 4.6 (range 1.8-7.6 U/L) and the oveall result of ADA in NTBM group was 7.2U/L \pm 8.2 (range 1.8-49.1 U/L). In TBM highest ADA was found $65.2 \mathrm{U} / \mathrm{L}$ and lowest was $1.0 \mathrm{U} / \mathrm{L}$; in NTBM the highest ADA was $49.1 \mathrm{U} / \mathrm{L}$ and lowest ADA was 1.8 $\mathrm{U} / \mathrm{L}$.(Table VII).

Table -VIII

Performance of CSF ADA activity

\begin{tabular}{lccc}
\hline & \multicolumn{2}{c}{ AFB culture } & \\
ADA category & Positive & Negative & Total \\
\hline ADA & $9(81.8 \%)$ & $17(34.7 \%)$ & $26(43.3 \%)$ \\
$>7.6 \mathrm{U} / \mathrm{L}$ & $($ True Positive) & (False Positive) & (all ADA positive) \\
ADA & $2(18.2 \%)$ & $32(65.3 \%)$ & $34(56.7 \%)$ \\
$<7.6 \mathrm{U} / \mathrm{L}$ & $($ False Negative) & (True Negative) & (all ADA negative) \\
\hline Total & $11(100 \%)$ & $49(100 \%)$ & $60(100 \%)$ \\
& (all CS positive) & (all CS Negative) & (Grand total) \\
\hline
\end{tabular}

Table -IX

Common tools of the performance of ADA activity:

\begin{tabular}{lc}
\hline Parameter & Result \\
\hline Sensitivity(SEN) & $81.82 \%$ \\
Specificity(SPE) & $65.31 \%$ \\
Accuracy & $68.33 \%$ \\
Positive predictive value (PPV) & $34.62 \%$ \\
Negative predictive value (NPV) & $94.12 \%$ \\
Postive Likelihood ratio (LR+) & 2.3 \\
NegativeLikelihood ratio (LR-) & 0.2 \\
\hline
\end{tabular}


Table $\mathrm{X}$ shows the performance of ADA activity to diagnose tuberculous meningitis. The true positive cases were $81.8 \%(9 / 11)$ and false negative were $18.2 \%(2 / 11)$; on the other hand the true negative cases were $65.3 \%$ (32/49) cases and false positive cases were $34.7 \%(17 / 49))$. Total ADA positive was $43.3 \%(26 / 60)$ and total negative was $56.7 \%(34 / 60)$ among the 60 cases.

Table IX shows the various tools of performance of ADA activity: the ADA sensitivity was $81.82 \%$,specificity $65.31 \%$,accuracy $68.33 \%$, PPV $34.62 \%$,NPV 94.12\%, positive likelihood ratio 2.3 and lastly negative liklihood ratio 0.2 .

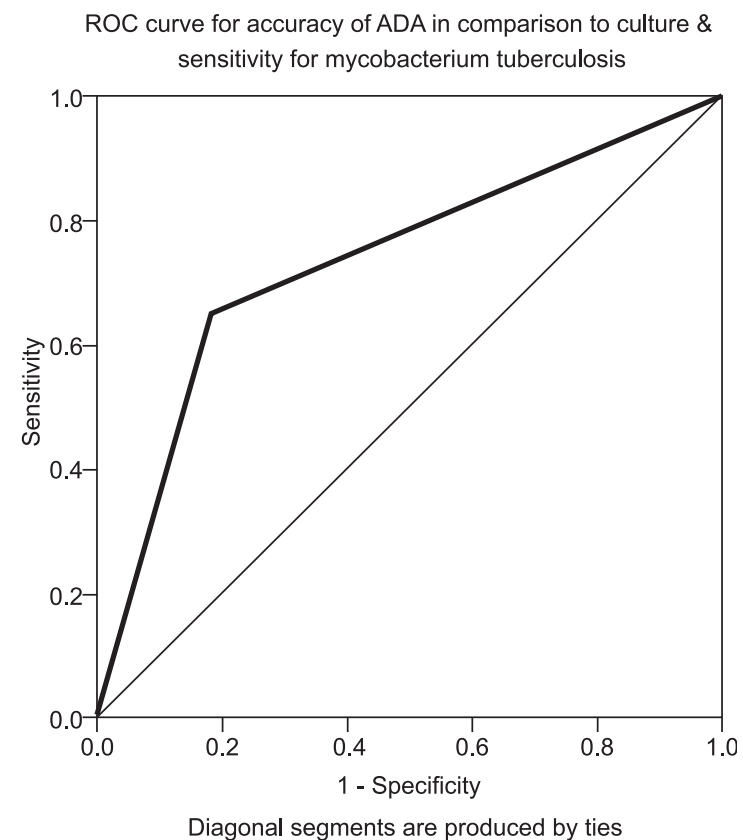

Fig.-4: ROC curve for detecting accuracy of $A D A$ in diagnosis of tuberculous meningitis

ROC curve for accuracy of ADA in comparison to culture sensitivity for mycobacterium tuberculosis.

Area Under the Curve

$\begin{array}{cccc}\text { Area } & \text { Std. Error } & \text { P Value } & 95 \% \mathrm{Cl} \\ .736 & .080 & .015 & 0.579-0.893\end{array}$

Test Result Variable(s): ADA category

Figure 4 shows ROC curve for detecting accuracy of ADA in diagnosis of tuberculous meningitis;Area under curve of .736 suggests a moderate accuracy of the test in detection of tubercular meningitis and Std. Error .080, P Value .015, 95\% Cl, 0.579 - 0.893.

\section{Discussion:}

The present study was done in 30 cases (TBM) and 30 control (NTBM). However,among the 30 TBM cases, one case was from NTBM group as intially diagnosed as pyogenic meningitis but latter on due to lack of antibiotic response and repeat CSF showed featues of TBM, it was included as TBM.Similarly two clinically suspected TBM cases were lastly diagnosed as meningitis due to lymphoma and another as Wilson's disease. So, these two cases were excluded from the study based on exclusion criteria. Sixty patients comprised 30 TBM, and 30 NTBM were selected that is similar to Rajendra et al ${ }^{11}$ who took 56 cases comprised tuberculous meningitis:29, pyogenic meningitis : 15 , and aseptic meningitis : 12 cases. The peak incidence in the present study was found in young adults in the age group of $20-39 y e a r s ~(53.3 \%)$. It is similar to another study where observed the peak incidence was $43 \%^{12}$. According to the present study, the incidence in male was $56.7 \%$ and in female $43.3 \%$. The incidence in both males and females is consistent with the study done by Gambhir et al. ${ }^{13}$. The reasons for the high occurance of infection in male might be due to the fact that being the main earning member of the family; they have to go outside and thus are more exposed and more chances of getting infections. Moreover females are reluctant to come to the hospital for treatment.

In the present study, history of fever is present in most of the cases (91.7\%). It was low-grade, more in the evening, associated with night sweats. In other study the incidence of fever was $87 \%$ and $58.9 \%(14,15)$.Fever was absent in about $10 \%$ cases; litrature told that fever can be absent in upto $25 \%$ of patients ${ }^{16}$. Seizures of generalized tonic and clonic type were noted in $8.3 \%$ of the cases of both TBM and NTBM groups. In one study, the incidence of seizures was $12.1 \%{ }^{15}$. The signs of meningeal irritation were present as neck rigidity in $95 \%$, Kernig's sign in $46.7 \%$.In another study, neck rigidity was $54 \%$ and Kernig's sign was $40 \%$ cases (Khatua et al., 1961) and on the other hand, neck rigidity was $100 \%$ in another study (Ramkrishna et al 2006). However in neurology text book, the overall meningeal signs is mentioned as $70 \%$ (Ashok,2008) 
The present study revealed that cranial nerve palsies were observed in $10 \%(6 / 60)$ of the cases, which is consistent with the other study and that was observed in 15.4\% (Venkataraman et al.,1980) cases. The commonnest was 6 th $50 \%(3 / 6)$, then 7 th $16.6 \%(1 / 6$ case) and one was third cranial nerve palsy ;another one case was with multiple cranial nerve palsy (vi,vii,ix,x,xi,xii). In one study it was found $6^{\text {th }}$ nerve palsy in $7.2 \%$ cases $^{15}$ and also reported $3.2 \%$ cases with isolated facial nerve palsies ${ }^{18}$. In the present study, the incidence of papilloedema was $13.3 \%(8 / 60)$ and optic atrophy $3.3 \%(2 / 60)$ of cases and all are in TBM group. Other study observed papilloedema in $16.1 \%$ of cases ${ }^{18}$. In the present study, limb weakness was noted in $20 \%$ (12/ $60)$ cases, higher rate of observation was also found and the limb weakness was in $37.9 \%$ cases $^{18}$ but literaure review shows focal neurological findings was in $16-18 \%{ }^{19}$ that is more similar to present study. Similarly MT test shows higher rate of positivty in TBM patients and it was positive in $43.7 \%(13 / 30)$ cases.MT positive was found in 50\% cases of TBM in another study ${ }^{19}$.

The biochemical parameters of CSF in various meningitis showed some disimilarity between TBM and pyogenic meningitis for example CSF total cell count was higher in TBM than pyogenic meningitis. It was possibly due to getting partial treatment of pyogenic meningitis before coming to tertiary level hospital.Several case series have established CSF staining sensitivities of $<20 \%{ }^{20,21}$. However in the present study CSF ZN staning for AFB of all cases were negative.MTB culture studies in several case series established CSF culture sensitivities of 25 to $70 \%^{22,23}$ and in one study shows the sensitivity of AFB culture is $40 \%{ }^{24}$. In the present study $36.6 \%(11 / 30)$ cases were culture positive. Mean CSF ADA was higher in AFB positive cases than AFB negative cases, so there is a postive correlation between AFB and ADA.

In the interpretation of CSF-ADA levels for diagnosis of TBM two important points must be considered, one is the definition of TBM patients which may have a direct impact on the results of this interpretation and another is the value of cut-off which has a great importance in the evaluation of the sensitivity and specificity of the CSF-ADA test. The amount of this cut-off is controversial at the present time. Parsad et $a /{ }^{9}$ reported a sensitivity and specificity of $100 \%$ and $97.87 \%$ respectively with a 3.30IU/L cut-off value for ADA in the diagnosis of tuberculous meningitis. On the other hand, Chotmongkol et al ${ }^{3}$ in their study reported $75 \%$ sensitivity and $93 \%$ specificity for CSF-ADA level in diagnosis of TBM with a $15.5 \mathrm{I} \mathrm{U} / \mathrm{L}$ cut off and Kashyap et al. ${ }^{25}$ in their study reported that with a $11.39 \mathrm{IU} / \mathrm{L}$ cut-off, the sensitivity and specificity of ADA measurement in diagnosis of TBM in CSF samples of their patients were $82 \%$ and $83 \%$ respectively. Corral et a ${ }^{26}$ reported $57 \%$ sensitivity and $87 \%$ specificity with a $8.5 \mathrm{IU} / \mathrm{L}$ cut-off for CSFADA level in the diagnosis of TBM in HIV infected patients and Gambhir et al ${ }^{13}$ in their study reported the sensitivity and specificity of $85 \%$ and $88.0 \%$ respectively for CSF-ADA levels in diagnosis of TBM with a $6.97 \mathrm{IU} / \mathrm{L}$ cut off value. Baheti and his colleagues reported $95.85 \%$ sensitivity and $92.85 \%$ specificity for CSF-ADA test in differentiating tuberculous meningitis from non-tuberculous meningitis with a $6.5 \mathrm{IU} / \mathrm{L}$ cut-off. Baro et al. ${ }^{27}$ proposed a cut-off value of $6.5 \mathrm{U} / \mathrm{L} / \mathrm{min}$ and showed sensitivity of $83.3 \%$ and specificity of $88.3 \%$.

In this study, sensitivity and specificity of CSF-ADA level in comparison with MTB culture results in TBM diagnosis in 30 TBM patients with a $7.6 \mathrm{IU} / \mathrm{L}$ cut off were $81.82 \%$ and $65.31 \%$ respectively. Mean CSF ADA level was significantly higher in TBM patients (14.01 \pm 12.4$)$ as compared to NTBM $(7.2 \pm 8.2)$, $\mathrm{P}=0.01$.

In the present study it was apparent that the level of CSF ADA activity in TBM was significantly high compared to overall NTBM group, but high level(>7.6U/I) was also observed in other individual group particularly bacterial meningitis (14.01U/l vs $10.4 \mathrm{U} / \mathrm{l}$, TBM vs bacterial meningitis). The possible explanation is that the activity is usually higher in CSF which contains numerous cells. These findings are in agreement with other study where it was observed a high ADA activity in CSF obtained at the initial diagnostic lumbar puncture from bacterial meningitis cases ${ }^{27}$. It was also reported an overlap between TBM and non-TBM patients, especially for infectious neurological disorders like pyogenic meningitis ${ }^{13}$. It was also found that elevated levels 
of CSF ADA are not specific for TBM. Diseases like pyogenic meningitis, CNS lymphoma, and fungal meningitis were shown to have elevated CSFADA ${ }^{27}$.

On the basis of present result when ADA interpreted together with clinical signs and symptoms and other laboratory tests, it can be a useful adjunctive rapid test for the diagnosis of TBM. The estimation of ADA activity in CSF is therefore serves as relatively simple, inexpensive and reliable tool in the diagnosis of TBM and management especially when other clinical laboratory tests are negative within sensitive limit.This study along with Gautam et al.and kashyap et al studies reemphasized that CSF-ADA level measurement can be used as a good, rapid and reliable laboratory test for diagnosing tuberculous meningitis at least in high prevalence and in cases of the incidence of tuberculosis in low income regions.

\section{Conclusion:}

The study found the sensitivity of the test to be $81.82 \%$; specificity $65.31 \%$, positive predictive value $34.62 \%$ and negative predictive value $94.12 \%$, and so it can be concluded that ADA estimation in CSF is not only simple, inexpensive and rapid but also fairly specific method for making a diagnosis of tuberculous etiology in TBM, especially when there is a dilemma of differentiating the tuberculous etiology from non-tuberculous. For this reason CSF ADA estimation in TBM may find a place as a routine investigation.

\section{References:}

1. WHO reports 2003. Global TB control, surveillance, planning, financing; communicable diseases, WHO, Geneva p18.

2. Garcia-Monco JC, Marra CM, editor. CNS Tuberculosis. Neurologic Clinics. 1999; 17(4): 737-60.

3. Chotmongkol $\mathrm{V}$, Jitpimolmard $\mathrm{S}$ and Thavornpitak Y. 'Corticosteroid in tuberculous meningitis', J Med Assoc Thai. 1996; 79: 83-90.

4. Zuger A, Lowy FD. Tuberculosis of the central nervous system. In: Scheld WM, Whitley
RJ,Durack DT, eds. Infections of the central nervous system. New York: Raven Press, 1991; pp- 425-56.

5. Sullivan JL, Osborne WR and Wedgewood RJ. 'Adenosine deaminase activity in lymphocytes', Br J Haematol. 1977; 37: 1578.

6. Rana SV, Singhal RK, Singh K and Kumar L. 'Adenosine deaminase levels in cerebrospinal fluid as a diagnostic test for tuberculous meningitis in children', Indian J Clinbiochem. 2004; 19: 5-9.

7. Kumar R, Singh SN, Kohlin. 'A diagnostic rule for tuberculosis meningitis', Archieves of the diseae of childhood. 1999; 81: 221-4.

8. Piras MA, Gakis C. 'Cerebrospinal fluid adenosine deaminase activity in tuberculousmeningitis',Enzyme. 1973; 14: 311-16.

9. Prasad R. Kumar A and Khanna BK. 'Adenosine deaminase activity in cerebrospinal fluid for diagnosis of tuberculous meningitis', Indian J Tub. 1991; 38: 99-102.

10. Ribera E, Martinez-Vazquez JM, Ocana I, Segura RM and Pascual C. 'Activity of adenosine deaminase in cerebrospinal fluid for the diagnosis and follow-up of tuberculous meningitis in adults', $J$ Infect Dis, 1987; 155: 603-7.

11. Rajendra Prasad and Anil Kumar, 'Adenosine deaminase activity cerebrospinal fluid for diagnosis tuberculous meningitis', Ind. J. Tub. 1991; 38: 99.

12. Ramkishna. Adenosine deaminase acitivity in cerebrospinal fluid for diagnosis of tubrculosis meningitis,www.scribd.com/doc/52584960/ ada in-tb-meningitis 2006.

13. Gambhir IS, Mehta M, Singh DS and Khanna HD. 'Evaluation of CSF-adenosine deaminase activity in tubercular meningitis', J Assoc physician India. 1999; 47: 192-4.

14. Khatua SP. 'Tuberculous meningitis in children: Analysis of 231 cases', J.Indian Med Ass. 1961; 37: 332. 
15. Virmani Vimla,Venkataraman S, Sarasavani, $\checkmark$ Rao, and Ahuja GK.'Clinical spectrum of neurotuberculosis in adults', Jr Asso Phys Ind . 1980; 28: 431-7.

16. Tarakad S Ramachandran. Tuberculous Meningitis; Chief Editor: Karen L Roos; Medscape; http://emedicine.medscape.com/ article/1166190. 2011.

17. Khatua SP. 'Tuberculous meningitis in children: Analysis of 231 cases', J.Indian Med Ass. 1961; 37: 332.

18. Venkataraman S, Sarasavani, V Rao, Ahuja GK and VimlaVirmani,1980, 'Clinical spectrum of neurotuberculosis in adults', JrAssoPhys Ind. 1980; 28: 431-37.

19. Ashok Verma. Infections of the nervous system.In:WG Bradely, R B Darott,GM Fenidel,J Jancovic(eds).Neurology in Clinical Practice;5th edn, New York, Elsevier: 2008; 1420.

20. Tofte RW, Peterson PK, Kim Y, ' Opsonic activity in normal human cerebrospinal ûuid for selected bacterial species', Infect Immun. 1979; 26: 1093-8.

21. Sutlas $P$ N, A Unal, H Forta, S Senol, and D Kirbas, 'Tuberculous meningitis in adults: review of 61 cases', Infection. 2003; 31: 387-91.
22. Kent S J, S M Crowe, A Yung, C R Lucas and A M Mijch. 'Tuberculous meningitis: a 30-year review', Clin. Infect. Dis. 1993; 17: 987-94.

23. Naughten $E, A M$ Weindling, $R$ Newton, and $B$ $D$ Bower. 'Tuberculous meningitis in children. Recent experience in two English centres', Lancet 1981; ii: 973-5.

24. Ogawa S K, M A Smith, D J Brennessel and F $D$ Lowy. 'Tuberculous meningitis in an urban medical center', Medicine (Baltimore.)v 1987; 66: 317-26.

25. Kashyap RS, Kainthla RP and Mudaliar AV. 'Cerebrospinal fluid adenosine deaminase activity: a complimentary tool in the early diagnosis of tuberculous meningitis', Cerebrospinal Fluid Res. 2006; 30: 3-5.

26. Corral I, C Quereda, E Navas, P Martin-Davila, M J Perez-Elias, J L Asado et al,2004. 'Adenosine deaminase activity in cerebrospinal fluid of HIV-infected patients: limited value for diagnosis of tuberculous meningitis', Eur. J. Clin. Microbiol. Infect. Dis. 2004; 23: 471-76.

27. Baro M, Acevedo L, Lagos ME. 'Usefulness of adenosine deaminase determination in cerebrospinal fluid for the diagnosis of meningeal tuberculosis; 4 years' experience at a public hospital'. Rev Med Chill, 1996; 124: 319-26. 